\title{
Probabilistic integrity assessment of turbine disks in presence of potential defects
}

\author{
Stefano Beretta ${ }^{1, a}$, Stefano Foletti ${ }^{1}$, Mauro Madia ${ }^{2}$ and Emanuela Cavalleri ${ }^{3}$ \\ ${ }^{1}$ Politecnico di Milano, Dipartimento di Meccanica, 20156 Milano, Italy \\ ${ }^{2}$ Federal Institute for Materials Research and Testing (BAM), Division 9.1, 12205 Berlin, Germany \\ ${ }^{3}$ Ansaldo Energia, 16152 Genova, Italy
}

\begin{abstract}
Rotor disks for gas turbines are heavy components and are usually designed following a safe-life approach, where the low-cycle fatigue analysis is carried out referring to design life curves with suitable probabilistic margins. However, in the case of such a heavy component there is the possibility of rare occurrence of undetected defects or, better, the need to identify the defect acceptability for the different rotor regions (considering stress, temperature, mission profile). In order to carry out such calculations for a turbine rotor disk a software named \{AStrID\} (Assessment of the Structural Integrity of Disks) has been developed in close cooperation between Politecnico di Milano and Ansaldo Energia (AEN). This paper summarizes its background and some of the relevant features.
\end{abstract}

\section{Introduction}

Accidents involving rotating equipment such as steam and gas turbines can result also in damage of other components in plants and loss of human lives. In order to minimize accidents it is very important to understand and prevent failures. The type of failure giving the highest contribution to the number of losses is represented by the burst of disks due to accidental overspeeds [1]. There are several approaches and methodologies in the literature which allow to carry out a probabilistic structural integrity assessment specifically for rotating components $[2,3]$.

The software AStrID has been developed with the goal to have a reliable tool for the thorough and automatic assessment of turbine disks, consider the prospective occurrence of an overspeed at the end of the prospective service life $N_{\text {life }}$ of the order of 3000-5000 cycles. Among the features of the software, the three most important capabilities are the following: i) residual lifetime after a prospective defect with size $a_{o}$ has been detected; ii) calculation of the maximum permissible defect $a_{p}$ given a target residual life $N_{\text {life }}$. The CDF (Crack Driving Force) approach has been implemented in the software for calculating failure probability at overspeed, according to SINTAP/FITNET [4]. CDF is typically expressed in terms of a plastic-corrected stress intensity factor $K_{J}$, and failure occurs in the critical

\footnotetext{
${ }^{a}$ Corresponding author: stefano.beretta@polimi.it

This is an Open Access article distributed under the terms of the Creative Commons Attribution License 4.0, which permits unrestricted use, distribution, and reproduction in any medium, provided the original work is properly cited.
} 


\section{MATEC Web of Conferences}
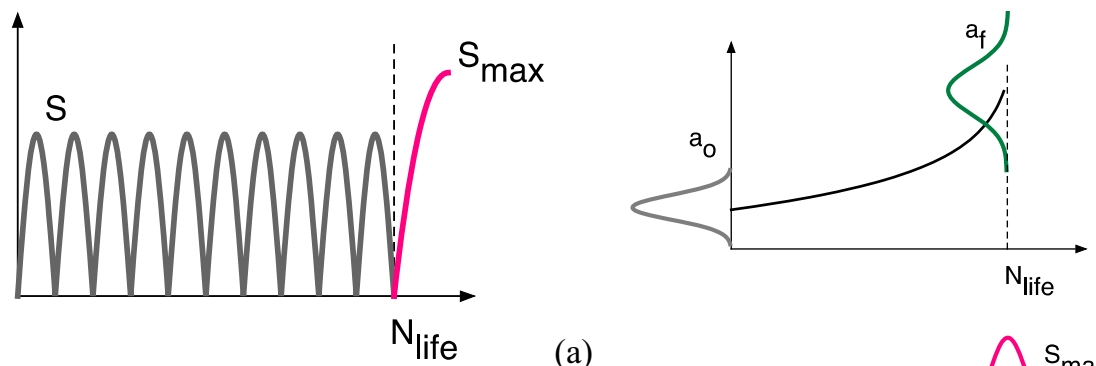

(a)
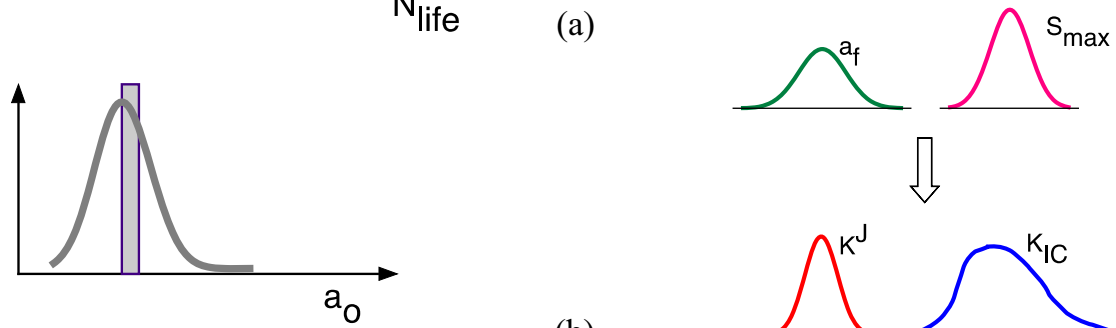

(b)

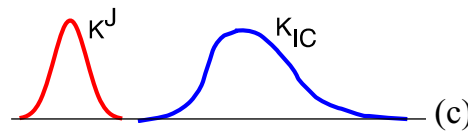

Figure 1. Scheme of the calculations: a) service of a turbine and assessment against a prospective overspeed; b) sizing error related to a prospective NDT; c) scheme of calculation of failure probability.
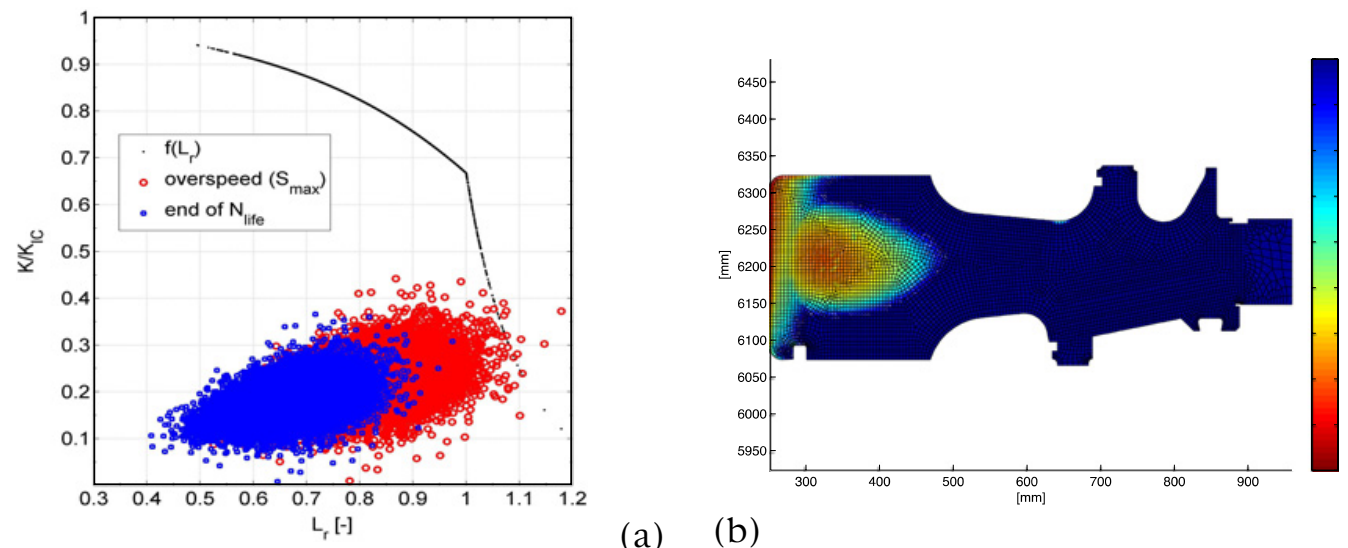

(a)

(b)

Figure 2. Probabilistic calculations in ASTRID: a) Montecarlo simulation according to Fig. 1.c; b) map of the permissible defect in a disk for obtaining a failure probability of $5 \times 10^{-5}$ considering $N_{\text {life }}$ turbine start-ups.

condition for the component when:

$$
\mathrm{K}^{\mathrm{J}} \geq \mathrm{K}_{\mathrm{mat}}(\mathrm{T})
$$

\section{Probabilistic assessment}

The probabilistic asseesment onto a FAD diagram is shown in Fig. 2. The nature of the software AStrID is semi-probabilistic in the sense that the structural integrity assessment is carried out with deterministic values adopting Partial Safety Factors-PSF- which have been tuned with Montecarlo simulations in order to achieve a target failure probability of $5 \times 10^{-5}$ at the end of the prospective life. 


\section{References}

[1] Nelson, W. E., and Monroe, P. C. In 26th Turbomachinery Symposium, pp. 129-142 (1996)

[2] Leverant, G., Millwater, H., McClung, R., Enright, M., J. Engng Gas Turbines Power, 126 (2004)

[3] Wu, Y., Enright, M., and Millwater, H., AIAA Journal, 40 (2002)

[4] Zerbst, U. et al. Fitness-for-Service Fracture Assessment of Structures Containing Cracks A Workbook based on the European SINTAP/FITNET Procedure. Elsevier (2007) 